\title{
Probing the gravitational redshift with an Earth-orbiting satellite
}

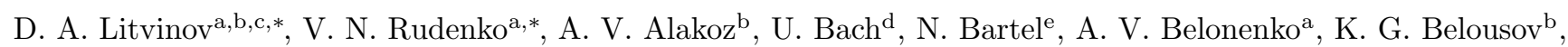
M. Bietenholz ${ }^{\mathrm{e}, \mathrm{f}}$, A. V. Biriukov ${ }^{\mathrm{b}}$, R. Carman ${ }^{\mathrm{g}}$, G. Cimóh,i ${ }^{\mathrm{h}}$, C. Courde ${ }^{\mathrm{j}}$, D. Dirkx ${ }^{\mathrm{k}}$, D. A. Duev ${ }^{\mathrm{l}}$, A. I. Filetkin ${ }^{\mathrm{a}}$, G. Granato ${ }^{\mathrm{k}}$, L. I. Gurvits ${ }^{\mathrm{h}, \mathrm{k}}$, A. V. Gusev ${ }^{\mathrm{a}}$, R. Haas ${ }^{\mathrm{m}}$, G. Herold ${ }^{\mathrm{n}}$, A. Kahlon ${ }^{\mathrm{o}}$, B. Z. Kanevsky ${ }^{\mathrm{b}}$, V. L. Kauts ${ }^{\mathrm{b}, \mathrm{c}}$, G. D. Kopelyansky ${ }^{\mathrm{b}}$, A. V. Kovalenko ${ }^{\mathrm{p}}$, G. Kronschnabl ${ }^{\mathrm{q}}$, V. V. Kulagin ${ }^{\mathrm{a}}$, A. M. Kutkin ${ }^{\mathrm{b}}$, M. Lindqvist ${ }^{\mathrm{m}}$,

J. E. J. Lovello, H. Mariey ${ }^{\mathrm{j}}$, J. McCallum ${ }^{\mathrm{o}}$, G. Molera Calvés ${ }^{\mathrm{h}, \mathrm{r}}$, C. Moore ${ }^{\mathrm{s}}$, K. Moore ${ }^{\mathrm{e}}$, A. Neidhardt ${ }^{\mathrm{t}}$, C. Plötz ${ }^{\mathrm{q}}$, S. V. Pogrebenko ${ }^{\text {, A. A Pollard }}$, N. K. Porayko ${ }^{\mathrm{a}, \mathrm{d}}$, J. Quick ${ }^{\mathrm{f}}$, A. I. Smirnov ${ }^{\mathrm{b}}$, K. V. Sokolovsky ${ }^{\mathrm{a}, \mathrm{b}, \mathrm{u}}$, V. A. Stepanyants ${ }^{\mathrm{v}}$, J.-M. Torre ${ }^{\mathrm{j}}$, P. de Vicente ${ }^{\mathrm{w}}$, J. Yang ${ }^{\mathrm{m}}$, M. V. Zakhvatkin ${ }^{\mathrm{v}}$

\author{
${ }^{a}$ Sternberg Astronomical Institute, Lomonosov Moscow State University, Universitetsky pr. 13,119991 Moscow, Russia \\ ${ }^{b}$ Astro Space Center, Lebedev Physical Institute, Profsoyuznaya 84/32, 117997 Moscow, Russia \\ ${ }^{c}$ Bauman Moscow State Technical University, 2-ya Baumanskaya 5, 105005 Moscow, Russia \\ ${ }^{d}$ Max-Planck-Institut für Radioastronomie, Auf dem Hügel 69, 53121 Bonn, Germany \\ e York University, Toronto, Ontario M3J 1P3, Canada \\ ${ }^{f}$ Hartebeesthoek Radio Astronomy Observatory, P.O. Box 443, Krugersdorp 1740, South Africa \\ ${ }^{g}$ Yarragadee Geodetic Observatory, Geoscience Australia, PO Box 137 Dongara 6525, Western Australia \\ ${ }^{h}$ Joint Institute for VLBI ERIC, PO Box 2, 7990 AA Dwingeloo, The Netherlands \\ ${ }^{i}$ ASTRON, the Netherlands Institute for Radio Astronomy, PO 2, 7990 AA Dwingeloo, The Netherlands \\ ${ }^{j}$ Université Côte d'Azur, CNRS, Observatoire de la Côte d'Azur, IRD, Géoazur, 2130 route de l'Observatoire, O6460 Caussols, France \\ ${ }^{k}$ Department of Astrodynamics and Space Missions, Delft University of Technology, 2629 HS Delft, The Netherlands \\ ${ }^{l}$ California Institute of Technology, Pasadena, CA 91125, USA \\ ${ }^{m}$ Department of Space, Earth and Environment, Chalmers University of Technology, Onsala Space Observatory, 439 92 Onsala, Sweden \\ ${ }^{n}$ Geodetic Observatory Wetzell (GOW), Germany \\ ${ }^{\circ}$ School of Physical Sciences, University of Tasmania, Private Bag 37, 7001 Hobart, Australia \\ ${ }^{p}$ Pushchino Radio Astronomy Observatory, Astro Space Center, Lebedev Physical Institute, 142290 Pushchino, Russia \\ ${ }^{q}$ Federal Agency for Cartography and Geodesy, Sackenrieder Str. 25, D-93444 Bad Kötzting, Germany \\ ${ }^{r}$ Finnish Geospatial Research Institute, Geodeetinrinne 2, 02430 Masala, Finland \\ ${ }^{s}$ EOS Space Systems Pty Limited, Mt Stromlo Observatory, Cotter Road, Weston Creek, ACT 2611, Australia \\ ${ }^{t}$ Technical University of Munich, Geodetic Observatory Wettzell, Sackenrieder Str. 25, D-93444 Bad Kötzting, Germany \\ ${ }^{u}$ IAASARS, National Observatory of Athens, Vas. Pavlou \& I. Metaxa, 15236 Penteli, Greece \\ ${ }^{v}$ Keldysh Institute for Applied Mathematics, Russian Academy of Sciences, Miusskaya sq. 4, 125047 Moscow, Russia \\ ${ }^{w}$ Observatorio de Yebes (IGN), Apartado 148, 19180 Yebes, Spain
}

\section{Abstract}

We present an approach to testing the gravitational redshift effect using the RadioAstron satellite. The experiment is based on a modification of the Gravity Probe A scheme of nonrelativistic Doppler compensation and benefits from the highly eccentric orbit and ultra-stable atomic hydrogen maser frequency standard of the RadioAstron satellite. Using the presented techniques we expect to reach an accuracy of the gravitational redshift test of order $10^{-5}$, a magnitude better than that of Gravity Probe A. Data processing is ongoing, our preliminary results agree with the validity of the Einstein Equivalence Principle.

Keywords: RadioAstron, gravitational redshift, Equivalence Principle, atomic clocks

\section{Introduction}

Quantum theory and general relativity are the two pillars of modern physics. However, they are incompatible. Attempts to quantize gravitation in the frameworks of string theory or loop quantum gravity inevitably lead to a violation of the Einstein Equivalence Principle (EEP)

\footnotetext{
*Corresponding author at: Sternberg Astronomical Institute, Lomonosov Moscow State University, Universitetsky pr. 13, 119991 Moscow, Russia.

Email addresses: litvirq@yandex.ru (D. A. Litvinov), rvn@sai.msu.ru (V. N. Rudenko)
}

and thus to a breakdown of the metric nature of gravitation 1. Although there exist attempts to preserve the unquantized status of gravitation, they have not created a compelling case so far 2. Tests of the EEP are therefore of primary interest to characterize any unified theory of interactions.

Much progress in the field of EEP tests was made with direct involvement and under the leadership of V. B. Braginsky, the founder of a gravitational physics school (see, e.g. [3, 4, 5]). With many coauthors of this paper proudly regarding Vladimir Borisovich as a teacher, we would like to dedicate this work to his memory. 
In this paper we present an approach to probing the gravitational redshift effect, which constitutes a test of the Local Position Invariance aspect of the EEP, using the RadioAstron satellite 6]. A highly eccentric orbit and an ultra-stable on-board atomic hydrogen maser clock make RadioAstron a unique laboratory for such test. The idea of experiments of this kind is to compare the rate of time flow at different space-time points against the gravitational potential difference between them. In the simplest case when time is measured by identical clocks and the gravitational field is weak, the basic equation reads [1]:

$$
\frac{\Delta T}{T}=(1+\varepsilon) \frac{\Delta U}{c^{2}},
$$

where $\Delta T / T$ is the fractional difference of time intervals measured by the clocks, $\Delta U$ is the gravitational potential difference between them, $c$ is the speed of light, and $\varepsilon$ is the violation parameter to be determined. In unified theories $\varepsilon$ is usually non-zero and depends on the clock type and element composition of the gravitational field source, while in general relativity and any other metric theory of gravitation $\varepsilon=0$.

The concept of a satellite-based gravitational redshift experiment was developed and realized by Vessot et al. in the suborbital Gravity Probe A (GP-A) mission [7], which yielded the best such test to date: it found $\varepsilon=$ $(0.05 \pm 1.4) \times 10^{-4}$ (for hydrogen maser clocks), with $\delta \varepsilon=$ $1.4 \times 10^{-4}$ constituting the accuracy of the test $(1 \sigma)$. A modified approach we have developed for RadioAstron allows us, in principle, to reach an accuracy of $\delta \varepsilon \sim 10^{-6}$, benefitting from a better performing hydrogen maser $(\mathrm{H}-$ maser) and prolonged data accumulation 8]. However, technical and operational constraints discussed below limit the achievable accuracy to $\delta \varepsilon \sim 10^{-5}$. Several competing experiments are currently at various stages of preparation or realization, with accuracy goals ranging from $4 \times 10^{-5}$ to $2 \times 10^{-6}$ 9, 10, 11 .

The RadioAstron project is an international collaborative mission centered around the $10-\mathrm{m}$ space radio telescope, with the primary goal of performing Space VLBI (Very-Long-Baseline Interferometry) observations of celestial radio sources of different nature with an extraordinary high angular resolution [6]. The RadioAstron spacecraft is on a highly eccentric orbit around the Earth, evolving due to the gravitational influence of the Moon, as well as other factors, within a broad range of the orbital parameter space (perigee altitude 1,000 - 80,000 km, apogee altitude $270,000-370,000 \mathrm{~km})$. The gravitational redshift experiment is a part of the RadioAstron mission's Key Science Programme. The essential characteristic of the mission, making it suitable for the experiment, is the presence of the space-qualified H-maser VCH-1010 aboard the spacecraft 12 .

The outline of the paper is as follows. In Section 2 we present our approach to testing the gravitational redshift effect with RadioAstron, emphasizing similarities and differences between our Doppler compensation scheme and

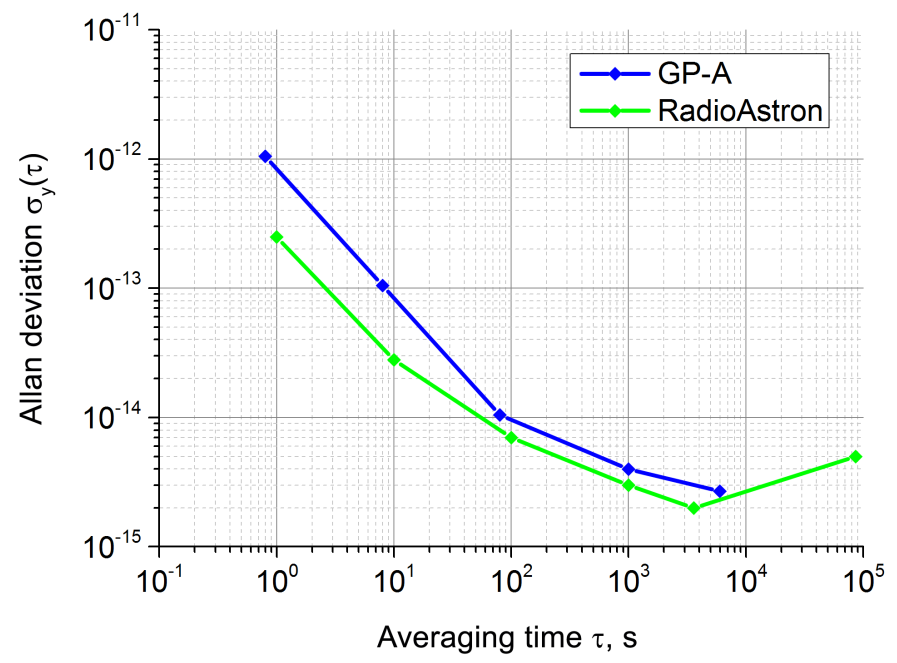

Figure 1: Comparison of the frequency stability of the RadioAstron VCH-1010 12 and GP-A VLG-10 13. H-masers in terms of the Allan deviation.

that of Gravity Probe A. In Section 3 we briefly discuss our data processing algorithms and describe how we treat small effects that are not cancelled by the Doppler compensation scheme. In Section 4 we give details of the measurements performed so far. We conclude with Section 5 by discussing the preliminary results and prospects for future research.

\section{Outline of the RadioAstron gravitational red- shift experiment}

There exist two approaches to testing the gravitational redshift effect in the field of the Earth. The first one is based on measuring the total value of the gravitational redshift between a ground station and a satellite. This approach, to be pursued by the ACES mission [10] and often called the absolute gravitational redshift measurement, is feasible only with accurate clocks. The second approach, pioneered by Gravity Probe A, requires a stable clock, such as an H-maser, and is based on measuring the modulation of the redshift effect caused by the spacecraft's motion along an eccentric orbit around the Earth. We follow the second approach, benefiting from the high stability of RadioAstron's H-maser (Fig. 1) and the deep modulation of the redshift effect due to the high eccentricity of the orbit (Fig. 2). The modulation approach has an important advantage over the absolute measurement - it eliminates most systematic errors and provides for statistical averaging of the results. The ultimate goal of the experiment, in either case, is to determine the EEP violation parameter $\varepsilon$ by comparing the experimentally measured redshift, $\Delta T_{\text {grav }}$ or $\Delta f_{\text {grav }}$, against the computed gravitational potential difference, $\Delta U$, between the ground and space-borne clocks using Eq. (1) or (2) (below).

In the gravitational redshift experiment with RadioAstron we detect the frequency change of RadioAstron's on- 


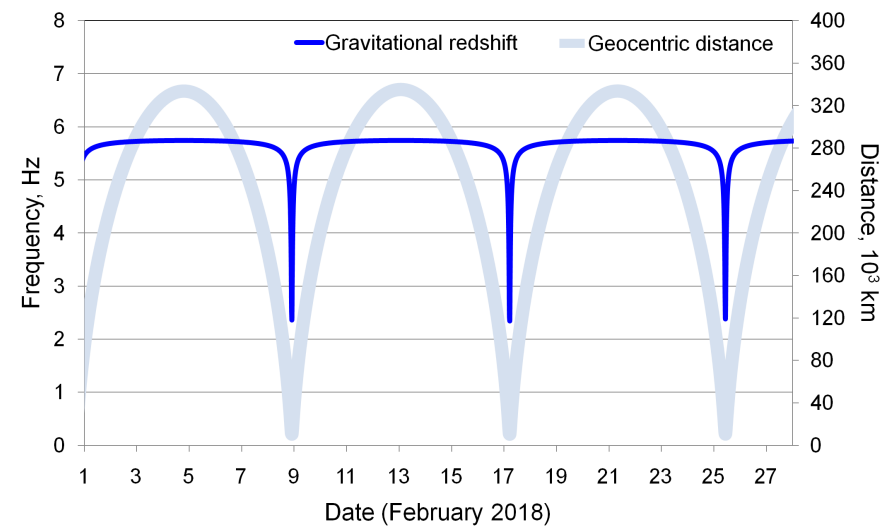

Figure 2: Variation of the gravitational frequency shift of the $8.4 \mathrm{GHz}$ downlink signal along the orbit during a low perigee epoch.

board H-maser due to gravitation by comparing it, with the help of radio links, with an H-maser at a ground station. The fractional frequency shift due to gravitation, $\Delta f_{\text {grav }} / f$, of a signal at frequency $f$ sent from the spacecraft to a ground station, is:

$$
\frac{\Delta f_{\mathrm{grav}}}{f}=(1+\varepsilon) \frac{\Delta U}{c^{2}},
$$

which reflects the same physics as Eq. (1). Either one of RadioAstron mission's dedicated tracking stations (TS), Pushchino (Moscow region, Russia) or Green Bank (West Virginia, USA), or a regular ground radio telescope (GRT) equipped with a 8.4 or $15 \mathrm{GHz}$ receiver may be used to receive the spacecraft signal. The small gravitational frequency shift, with a maximum value of $\Delta f_{\text {grav }} / f \sim 7 \times$ $10^{-10}$ at the apogee, needs to be extracted from a number of other effects influencing the signal sent from the spacecraft to the ground station [14]:

$$
\begin{aligned}
& \Delta f_{1 \mathrm{w}}= \\
& =f\left(-\frac{\dot{D}}{c}-\frac{v_{\mathrm{s}}^{2}-v_{\mathrm{e}}^{2}}{2 c^{2}}+\frac{\left(\mathbf{v}_{\mathrm{s}} \cdot \mathbf{n}\right)^{2}-\left(\mathbf{v}_{\mathrm{e}} \cdot \mathbf{n}\right) \cdot\left(\mathbf{v}_{\mathrm{s}} \cdot \mathbf{n}\right)}{c^{2}}\right) \\
& +\Delta f_{\text {grav }}+\Delta f_{\text {ion }}+\Delta f_{\text {trop }}+\Delta f_{\text {fine }}+\Delta f_{0}+O\left(\frac{v}{c}\right)^{3},
\end{aligned}
$$

where $\mathbf{v}_{\mathrm{s}}$ and $\mathbf{v}_{\mathrm{e}}$ are the velocities of the spacecraft and the ground station (in a geocentric inertial reference frame), $\dot{D}$ is the radial velocity of the spacecraft relative to the ground station, $\mathbf{n}$ is a unit vector in the direction opposite to that of signal propagation, $\Delta f_{\text {ion }}$ and $\Delta f_{\text {trop }}$ are the ionospheric and tropospheric shifts, $\Delta f_{\text {fine }}$ denotes various fine effects (phase center motion, instrumental, etc.), $\Delta f_{0}$ is the frequency bias between the ground and space $\mathrm{H}$ masers, and "1w" stands for "1-way" (space to ground).

There are two major problems in using Eq. (3) to determine $\Delta f_{\text {grav }}$ directly, at least for RadioAstron. First, the frequency bias, $\Delta f_{0}$, cannot be determined after launch

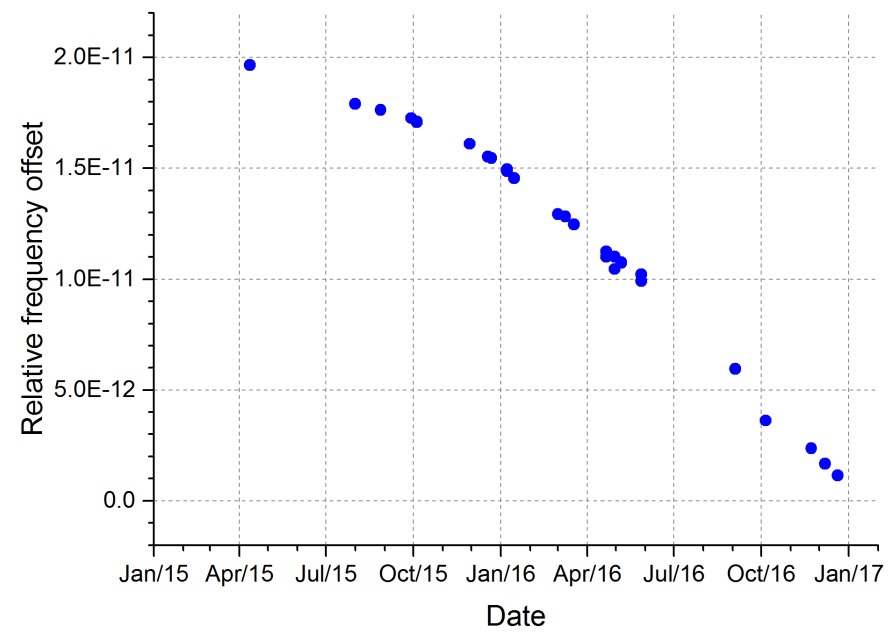

Figure 3: Frequency drift of the RadioAstron on-board H-maser relative to the $\mathrm{H}$-maser at the Green Bank TS.

without making use of Eq. 22, which requires the knowledge of $\varepsilon$. We do not expect $\Delta f_{0}$ to be negligible for $\mathrm{H}$-maser standards and, moreover, the well-known cavity "pulling" effect may cause it to drift over long times. We solve the bias problem by measuring only the modulation of the gravitational effect, $\Delta f_{\text {grav }}$, instead of its total value. In practice, this means having two or several observational sessions at greatly varying distances to the spacecraft. Although the measured value of the modulation of $\Delta f_{\text {grav }}$ is then free from the bulk of the bias, it still includes a contribution from the bias drift. The latter, however, may be determined from a series of observations at a constant geocentric distance. The drift measured this way (Fig. 3), indeed, turns out to be non-negligible $\left(3.6 \times 10^{-14} /\right.$ day now), so that we must take it into account.

The second problem with Eq. (3) is associated with the nonrelativistic Doppler shift, $-\dot{D} / c$. Since the range rate error $\delta \dot{D}$ is $\sim 2 \mathrm{~mm} / \mathrm{s}[15$, the error of the computed value of the Doppler shift is $\delta(\dot{D} / c) \sim 10^{-11}$, while $10^{-15}$ is required for achieving $\delta \varepsilon \sim 10^{-5}$. The first-order Doppler term, however, can be eliminated completely (for a TS), or its magnitude reduced sufficiently (for a GRT), owing to the availability of the 2-way ground-space-ground link (Fig. 4). The 2-way link signal is sent by a TS, received and phase-coherently retransmitted by the spacecraft, and finally received again by a TS and/or a GRT. The frequency shift of the 2-way link signal, for the simpler case of TS-space-TS propagation, is:

$$
\begin{aligned}
\Delta f_{2 \mathrm{w}}=f & \left(-2 \frac{\dot{D}}{c}-\frac{v_{\mathrm{s}}^{2}-v_{\mathrm{e}}^{2}}{c^{2}}+\frac{\left|\mathbf{v}_{\mathrm{s}}-\mathbf{v}_{\mathrm{e}}\right|^{2}}{c^{2}}-2 \frac{\mathbf{a}_{\mathrm{e}} \cdot \mathbf{n}}{c} \Delta t\right. \\
+ & \left.2 \frac{\left(\mathbf{v}_{\mathrm{s}} \cdot \mathbf{n}\right)^{2}-\left(\mathbf{v}_{\mathrm{e}} \cdot \mathbf{n}\right) \cdot\left(\mathbf{v}_{\mathrm{s}} \cdot \mathbf{n}\right)}{c^{2}}\right) \\
& +2 \Delta f_{\text {trop }}+2 \Delta f_{\text {ion }}+O(v / c)^{3},
\end{aligned}
$$

where $\mathbf{a}_{e}$ is the ground station acceleration and $\Delta t$ is the signal's light travel time 14. (A physically similar but calculationally more complex equation holds for the case of 
the 2-way link signal received by a nearby GRT.) Combining the 1-way (3) and 2-way (4) frequency measurements, we obtain:

$$
\begin{aligned}
\Delta f_{1 \mathrm{w}}-\frac{\Delta f_{2 \mathrm{w}}}{2} & =\Delta f_{\text {grav }}+f\left(-\frac{\left|\mathbf{v}_{\mathrm{s}}-\mathbf{v}_{\mathrm{e}}\right|^{2}}{2 c^{2}}+\frac{\mathbf{a}_{\mathrm{e}} \cdot \mathbf{n}}{c} \Delta t\right) \\
& +\Delta f_{0}+\Delta f_{\text {ion }}^{\text {(res) }}+\Delta f_{\text {fine }}+O(v / c)^{4}, \quad(5)
\end{aligned}
$$

where $\Delta f_{\text {ion }}^{(\text {res })}$ is the residual ionospheric shift (fully suppressed only for equal up- and downlink frequencies) and $\Delta f_{\text {fine }}$ denotes several "fine" effects, such as those due to the relativistic kinematic terms of order $(v / c)^{3}$ :

$$
\begin{gathered}
\frac{\Delta f^{(3)}}{f}=\frac{\mathbf{n} \cdot\left(\mathbf{v}_{\mathrm{e}}-\mathbf{v}_{\mathrm{s}}\right)}{c^{3}}\left(\Delta U-\frac{\left|\mathbf{v}_{\mathrm{s}}-\mathbf{v}_{\mathrm{e}}\right|^{2}}{2}+\left(\mathbf{a}_{\mathrm{e}} \cdot \mathbf{n}\right) c \Delta t\right) \\
+\frac{D}{c^{3}}\left(-\mathbf{v}_{\mathrm{s}} \cdot \mathbf{a}_{\mathrm{e}}-\left(\mathbf{j}_{\mathrm{e}} \cdot \mathbf{n}\right) c \Delta t+2 \mathbf{v}_{\mathrm{e}} \cdot \mathbf{a}_{\mathrm{e}}+\mathbf{v}_{\mathrm{e}} \cdot \nabla U_{\mathrm{e}}\right), \quad(6)
\end{gathered}
$$

where $\mathbf{j}_{\mathrm{e}}=\dot{\mathbf{a}}_{\mathrm{e}}$ is the ground station jerk and $\nabla U_{\mathrm{e}}$ is the gradient of the gravitational potential at the ground station location (see Section 3 for other fine effects). It is important to note that Eq. (5) is free from the nonrelativistic Doppler and tropospheric effects but retains the contribution of gravitation. The idea of the compensation scheme based on Eq. (5) was first realized in the GP-A mission, and the necessity of taking into account third-order kinematic effects was noted in [16, 17]. For RadioAstron, however, this scheme is not directly applicable because 1- and 2-way links cannot be operated simultaneously (Fig. 4). Nevertheless, two options for realizing the compensation scheme of Eq. (5) with RadioAstron have been devised.

The first option requires interleaving the 1-way " $\mathrm{H}$ maser" (Fig. 4 a) and 2-way "Coherent" (Fig. 4p) operation modes. The data recorded by GRTs (and the TS) contain only one kind of signal at any given time. However, if the switching cycle is short enough $(\sim 4 \mathrm{~min}$ at $8.4 \mathrm{GHz})$ we can interpolate the phases into the gaps with a corresponding frequency error of $\Delta f / f \sim 4 \times 10^{-15}$. Thus we obtain simultaneous frequency measurements of both kinds and can apply the compensation scheme of Eq. (5) to them directly. The approach based on interleaved measurements does not rely on any features of the signal spectrum and may be realized with telescopes equipped either with 8.4 or $15 \mathrm{GHz}$ receivers.

The second option for the Doppler compensation involves recording the $15 \mathrm{GHz}$ data downlink signal in the "Semi-Coherent" mode of synchronization of the on-board scientific and radio equipment [8], which is a kind of halfway between the 1-way "H-maser" and 2-way "Coherent" modes. In this mode the $7.2 \mathrm{GHz}$ uplink tone, the $8.4 \mathrm{GHz}$ downlink tone and the carrier of the $15 \mathrm{GHz}$ data downlink are phase-locked to the ground $\mathrm{H}$-maser signal, while the modulation frequency $(72 \mathrm{MHz})$ of the data downlink is phase-locked to the on-board H-maser signal (Fig. 44). This approach relies on the broadband $(\sim 1 \mathrm{GHz})$ nature of the $15 \mathrm{GHz}$ signal modulated using quadrature phase-shift keying (QPSK) and the possibility of turning its spectrum into a comb-like form by transmitting a predefined periodic data sequence (Fig. 5). As we have shown in [8, different subtones of the resulting spectrum act like separate links of the GP-A scheme and can be arranged in software postprocessing into a combination similar to that of Eq. (5), which is free from the 1st-order Doppler and tropospheric effects (the ionospheric term persists).

Despite some advantages of the second option from algorithmic and operational points of view, we give preference to the interleaved measurements approach as it provides for a larger number of participating GRTs due to the larger ground footprint of the on-board antenna at 8.4 $\mathrm{GHz}$ and wider availability of $8.4 \mathrm{GHz}$ receivers at GRTs.

\section{Data processing and fine effects}

The primary data for the experiment are the spacecraft signals at 8.4 and/or $15 \mathrm{GHz}$ received and recorded at a ground station. The majority of radio astronomy and geodetic radio telescopes are equipped with $\mathrm{H}$-maser standards and $8.4 \mathrm{GHz}$ receivers, enabling them to take part in the experiment. Recording of the spacecraft signal is performed in the ground H-maser timescale using standard VLBI back-end instrumentation. Initial data processing is based on the algorithms developed originally for PRIDE (Planetary Radio Interferometry and Doppler Experiment) [18 for recovering the phase of the received signal. Details of the algorithm and software modifications required to process interleaved data will be given in an upcoming publication [19. Here we briefly describe the approaches for correcting the recovered signal phases for a number of fine effects contributing to the right-hand side of Eq. (5):

- second- and third-order relativistic kinematic effects: computed from the orbital data (velocity determination accuracy of $\delta v \sim 2 \mathrm{~mm} / \mathrm{s}$ [15] is sufficient);

- gravitational potential difference between the spacecraft and the ground station: computed from the orbital data using the Earth gravitational potential model [20] (the position error of $\sim 200 \mathrm{~m}$ provided by radio ranging [15] is sufficient for distances $\gtrsim 40,000 \mathrm{~km}$, laser ranging required otherwise);

- residual ionospheric frequency shift: computed from 2 -frequency measurements (8.4 and $15 \mathrm{GHz}$ ), ionospheric total electron content (TEC) maps [21] and mapping functions [22], onsite GNSS receiver measurements;

- frequency shift due to the tidal gravitational field of the Sun and Moon [17: computed from the planetary and lunar ephemerides (JPL DE430);

- phase center motion of the on-board and tracking station antennas: computed from the orbital and housekeeping data 23]; 


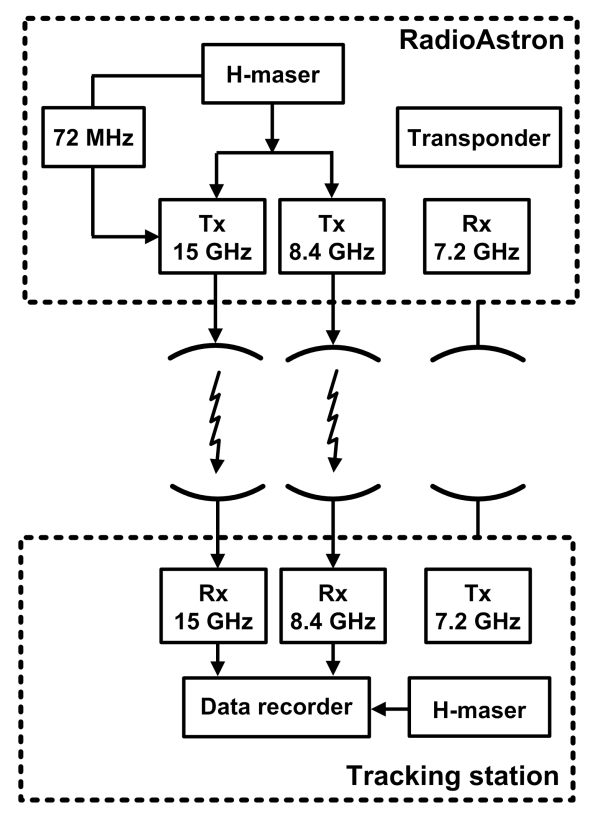

a)

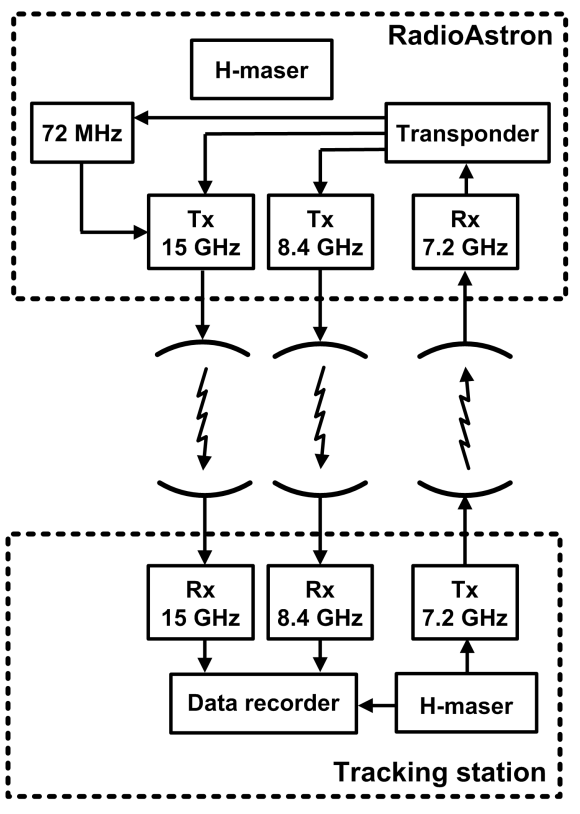

b)

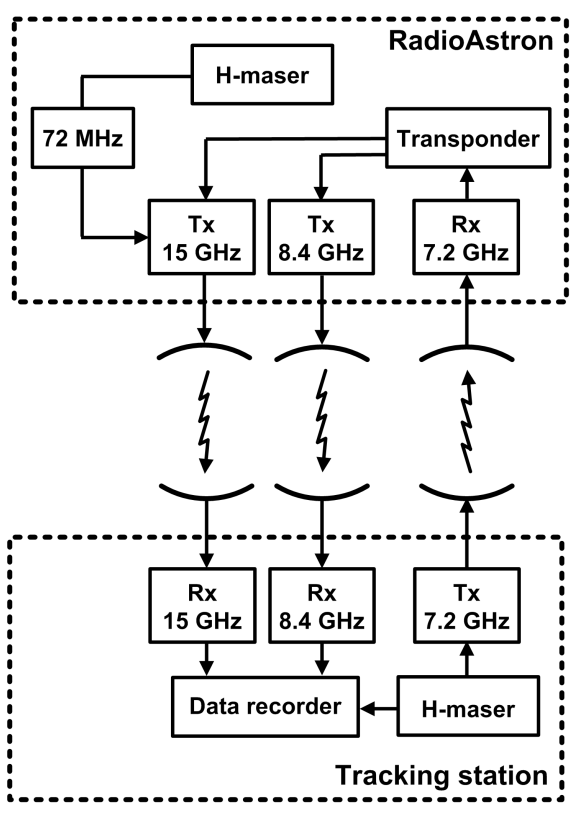

c)

Figure 4: Operation modes of the RadioAstron radio links: a) "H-Maser" (1-way); b) "Coherent" (2-way); c) "Semi-Coherent" (2-way).

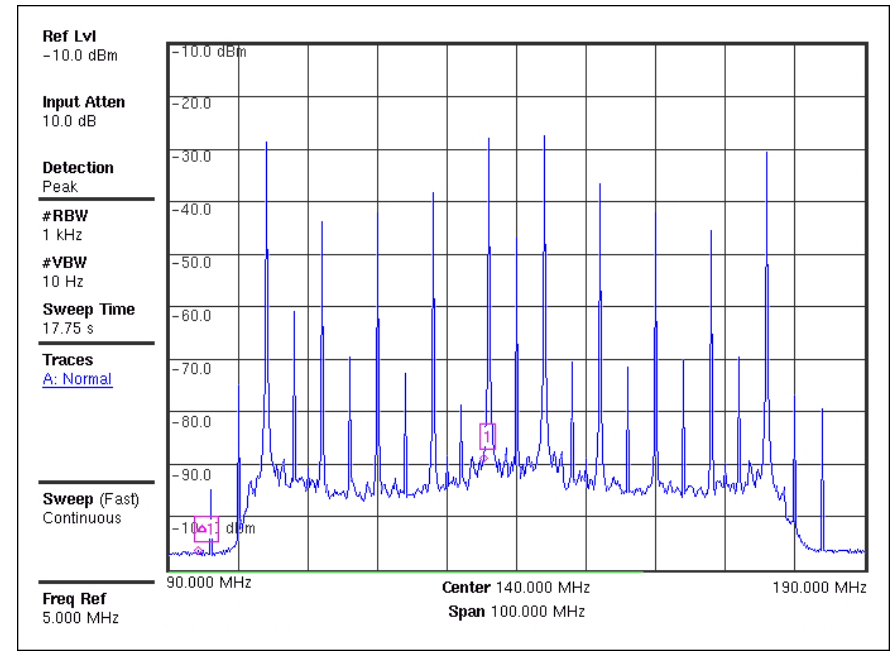

Figure 5: Spectrum analyser screenshot, showing the signal spectrum of the $15 \mathrm{GHz}$ data downlink in the "Test-2" mode of the on-board formatter.

- temperature dependence of the on-board H-maser: computed from the $\mathrm{H}$-maser sensitivity determined during ground tests and housekeeping data;

- magnetic field dependence of the on-board H-maser frequency: computed from the H-maser sensitivity determined during ground tests, the magnetic field model 24 and the orbital data;

- ground station motion due to solid Earth tides: computed from Earth models [25].

After the gravitational frequency shift has been measured experimentally in a series of observations at various distances on a single orbit, we fit it against the gravitational potential difference according to Eq. (2), thus obtaining a single measurement of $\varepsilon$. The accuracy of the result of $2-3$ years of planned data accumulation depends on the number of experiments performed and their parameters. Based on the experiment error budget 26 and taking into account the observations performed so far and those planned we expect the accuracy of the test to reach $\delta \varepsilon \sim 10^{-5}$.

\section{Measurements}

The observations for the experiment are limited both by the technical constraints of the RadioAstron satellite and competition for observational time with other science projects of the RadioAstron mission. The technical constraints are:

- the spacecraft's attitude limitations with respect to the Earth, Sun and Moon;

- the requirement that the spacecraft must be visible by the particular ground antenna;

- the requirement that the ground antenna must be within the on-board antenna's ground footprint.

A total of 18 experiments have been performed so far with the RadioAstron mission's Pushchino and Green Bank tracking stations supported by several European VLBI Network telescopes (Effelsberg, Onsala, Svetloe, Wettzell, Yebes, Zelenchukskaya), the Robert C. Byrd Green Bank Telescope, and several Very Long Baseline Array antennas. Extensive tests have been performed with the radio 


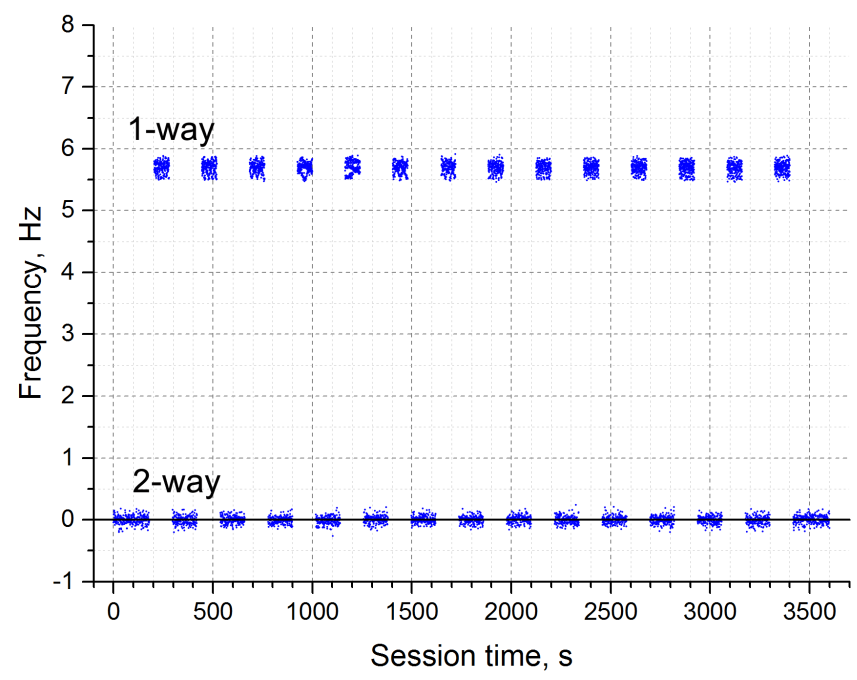

raks17an (28/05/2016, distance: $246,904-243,580 \mathrm{~km})$

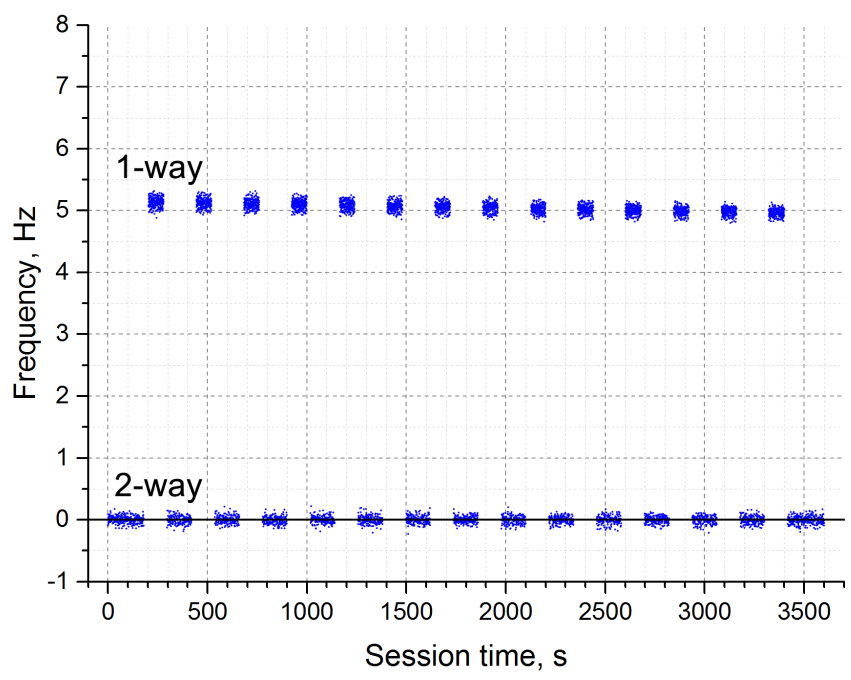

raks17ap (29/05/2016, distance: $54,414-42,171 \mathrm{~km})$

Figure 6: Residual frequencies of the 1- and 2-way 8.4 GHz signals measured with the Onsala 20-m telescope. The 1-way frequency residuals are not corrected for the gravitational redshift. This makes the variation of the gravitational frequency shift between the two sessions clearly visible (varying from $5.69 \mathrm{~Hz}$ to $4.96 \mathrm{~Hz}$ ).

telescopes and satellite laser ranging facilities of the Hartebeesthoek Radio Astronomy Observatory and AuScope VLBI Project's Yarragadee Observatory, which are to join the observations. All experiments were performed in the interleaved measurements mode (Fig. 6) and consisted of up to 4 observations, each $\sim 1$ hr long, distributed along the orbit over $\sim 20-50 \mathrm{hr}$. Most observations were accompanied by satellite laser ranging to guarantee an orbit determination accuracy at the cm-level [27]. The evaluation of our preliminary experimental results, which are consistent with $\varepsilon=0$, will be published elsewhere.

\section{Conclusions}

The RadioAstron satellite, with its highly eccentric orbit and on-board H-maser frequency standard, is a unique space-borne laboratory for probing the gravitational redshift effect, which constitutes a Local Position Invariance test of the Einstein Equivalence Principle. We have developed, and are implementing, a strategy for using the RadioAstron satellite to measure the gravitational redshift which takes into account the limitations of the spacecraft. We should be able to measure the redshift to an accuracy of $\sim 10^{-5}$, which is an order of magnitude better than the current best result of Gravity Probe A. Several measurements have already been obtained, and the data are being analyzed. Our preliminary results agree with the validity of the EEP. Some of the techniques we have developed, e.g. the particular realization of the Doppler compensation scheme, could be used for future space missions to test fundamental physics.

\section{Acknowledgements}

The authors wish to thank the team of the Astro Space Center of the Lebedev Physical Institute, and especially its head Nikolay Kardashev, and the RadioAstron project scientist Yuri Y. Kovalev, for constant support in preparation and realization of the experiment. The authors also thank the referees for valuable comments. Research for the RadioAstron gravitational redshift experiment is supported by the Russian Science Foundation grant 17-12-01488. The RadioAstron project is led by the Astro Space Center of the Lebedev Physical Institute of the Russian Academy of Sciences and the Lavochkin Scientific and Production Association under a contract with the Russian Federal Space Agency, in collaboration with partner organizations in Russia and other countries. The European VLBI Network is a joint facility of independent European, African, Asian, and North American radio astronomy institutes. Scientific results from data presented in this publication are derived from the following EVN project codes: EL053, EL057. The National Radio Astronomy Observatory is a facility of the National Science Foundation operated under cooperative agreement by Associated Universities, Inc. The Long Baseline Observatory is a facility of the National Science Foundation operated under cooperative agreement by Associated Universities, Inc. Hartebeesthoek Radio Astronomy Observatory is a facility of the South African National Research Foundation. AuScope Ltd is funded under the National Collaborative Research Infrastructure Strategy (NCRIS), an Australian Commonwealth Government Programme. 


\section{References}

[1] C. M. Will, The confrontation between general relativity and experiment Living Reviews in Relativity 17 (1) (2014) 4. doi: 10.12942/lrr-2014-4

URL http://dx.doi .org/10.12942/lrr-2014-4

[2] S. Carlip, Is quantum gravity necessary?, Classical and Quantum Gravity 25 (15) (2008) 154010. URL http://stacks . iop.org/0264-9381/25/i=15/a=154010

[3] V. B. Braginskii, V. I. Panov, Verification of the Equivalence of Inertial and Gravitational Mass, Soviet Journal of Experimental and Theoretical Physics 34 (1972) 463.

[4] V. Braginsky, V. Rudenko, Gravitational waves and the detection of gravitational radiation Physics Reports 46 (5) (1978) 165 - 200. doi:http://dx.doi.org/10.1016/0370-1573(78) 90192-8 URL http://www.sciencedirect.com/science/article/pii/ 0370157378901928

[5] V. B. Braginskii, V. N. Rudenko, Reviews of Topical Problems: Relativistic Gravitational Experiments, Soviet Physics Uspekhi 13 (1970) 165-181. doi:10.1070/PU1970v013n02ABEH004204

[6] N. S. Kardashev, V. V. Khartov, V. V. Abramov, V. Y Avdeev, A. V. Alakoz, Y. A. Aleksandrov, S. Ananthakrishnan, V. V. Andreyanov, A. S. Andrianov, N. M. Antonov, M. I. Artyukhov, M. Y. Arkhipov, W. Baan, N. G. Babakin, V. E. Babyshkin, N. Bartel', K. G. Belousov, A. A. Belyaev, J. J. Berulis, B. F. Burke, A. V. Biryukov, A. E. Bubnov, M. S. Burgin, G. Busca, A. A. Bykadorov, V. S. Bychkova, V. I. Vasil'kov, K. J. Wellington, I. S. Vinogradov, R. Wietfeldt, P. A. Voitsik, A. S. Gvamichava, I. A. Girin, L. I. Gurvits, R. D. Dagkesamanskii, L. D'Addario, G. Giovannini, D. L. Jauncey, P. E. Dewdney, A. A. D'yakov, V. E. Zharov, V. I. Zhuravlev, G. S. Zaslavskii, M. V. Zakhvatkin, A. N. Zinov'ev, Y. Ilinen, A. V. Ipatov, B. Z. Kanevskii, I. A. Knorin, J. L. Casse, K. I. Kellermann, Y. A. Kovalev, Y. Y. Kovalev, A. V. Kovalenko, B. L. Kogan, R. V. Komaev, A. A. Konovalenko, G. D. Kopelyanskii, Y. A. Korneev, V. I. Kostenko, A. N. Kotik, B. B. Kreisman, A. Y. Kukushkin, V. F. Kulishenko, D. N. Cooper, A. M. Kut'kin, W. H. Cannon, M. G. Larionov, M. M. Lisakov, L. N. Litvinenko, S. F. Likhachev, L. N. Likhacheva, A. P. Lobanov, S. V. Logvinenko, G. Langston, K. McCracken, S. Y. Medvedev, M. V. Melekhin, A. V. Menderov, D. W. Murphy, T. A. Mizyakina, Y. V. Mozgovoi, N. Y. Nikolaev, B. S. Novikov, I. D. Novikov, V. V. Oreshko, Y. K. Pavlenko, I. N. Pashchenko, Y. N. Ponomarev, M. V. Popov, A. Pravin-Kumar, R. A. Preston, V. N. Pyshnov, I. A. Rakhimov, V. M. Rozhkov, J. D. Romney, P. Rocha, V. A. Rudakov, A. Räisänen, S. V. Sazankov, B. A. Sakharov, S. K. Semenov, V. A. Serebrennikov, R. T. Schilizzi, D. P. Skulachev, V. I. Slysh, A. I. Smirnov, J. G. Smith, V. A. Soglasnov, K. V. Sokolovskii, L. H. Sondaar, V. A. Stepan'yants, M. S. Turygin, S. Y. Turygin, A. G. Tuchin, S. Urpo, S. D. Fedorchuk, A. M. Finkel'shtein, E. B. Fomalont, I. Fejes, A. N. Fomina, Y. B. Khapin, G. S. Tsarevskii, J. A. Zensus, A. A. Chuprikov, M. V. Shatskaya, N. Y. Shapirovskaya, A. I. Sheikhet, A. E. Shirshakov, A. Schmidt, L. A. Shnyreva, V. V. Shpilevskii, R. D. Ekers, V. E. Yakimov, Radioastron - a telescope with a size of $300000 \mathrm{~km}$ : Main parameters and first observational results Astronomy Reports 57 (3) (2013) 153194. doi:10.1134/S1063772913030025 URL http://dx.doi.org/10.1134/S1063772913030025

[7] R. F. C. Vessot, M. W. Levine, E. M. Mattison, E. L. Blomberg, T. E. Hoffman, G. U. Nystrom, B. F. Farrel, R. Decher, P. B. Eby, C. R. Baugher, Test of relativistic gravitation with a spaceborne hydrogen maser, Physical Review Letters 45 (1980) 20812084. doi:10.1103/PhysRevLett.45.2081

[8] A. V. Biriukov, V. L. Kauts, V. V. Kulagin, D. A. Litvinov, V. N. Rudenko, Gravitational redshift test with the space radio telescope "RadioAstron" Astronomy Reports 58 (11) (2014) 783-795. doi:10.1134/S1063772914110018 URL http://dx.doi .org/10.1134/S1063772914110018

[9] P. Delva, A. Hees, S. Bertone, E. Richard, P. Wolf, Test of the gravitational redshift with stable clocks in eccentric orbits: application to Galileo satellites 5 and 6 , Classical and Quantum Gravity 32 (23) (2015) 232003.

URL http://stacks . iop. org/0264-9381/32/i=23/a=232003

[10] M. P. Heß, L. Stringhetti, B. Hummelsberger, K. Hausner, R. Stalford, R. Nasca, L. Cacciapuoti, R. Much, S. Feltham, T. Vudali, B. Léger, F. Picard, D. Massonnet, P. Rochat, D. Goujon, W. Schäfer, P. Laurent, P. Lemonde, A. Clairon, P. Wolf, C. Salomon, I. Procházka, U. Schreiber, O. Montenbruck, The ACES mission: System development and test status, Acta Astronautica 69 (2011) 929-938. doi:10.1016/j. actaastro.2011.07.002

[11] P. Jetzer, General relativity tests with space clocks in highly elliptic orbits International Journal of Modern Physics D 26 (05) (2017) 1741014. arXiv:http://www. worldscientific.com/doi/pdf/10.1142/S0218271817410140 doi:10.1142/S0218271817410140

URL http://www.worldscientific.com/doi/abs/10.1142/ S0218271817410140

12] Active on-board hydrogen maser for Radioastron space mission VCH-1010, https://www.vremya-ch.com/english/product/ index6e49.html?Razdel=8\&Id=39, accessed: 2017-08-03.

[13] L. L. Smarr, R. F. C. Vessot, C. A. Lundquist, R. Decher, T. Piran, Gravitational waves and red shifts: A space experiment for testing relativistic gravity using multiple time-correlated radio signals General Relativity and Gravitation 15 (2) (1983) 129163. doi:10.1007/BF00762473 URL http://dx.doi.org/10.1007/BF00762473

[14] R. F. C. Vessot, M. W. Levine, A test of the equivalence principle using a space-borne clock, General Relativity and Gravitation 10 (1979) 181-204. doi:10.1007/BF00759854

[15] G. S. Zaslavskiy, M. V. Zakhvatkin, V. A. Stepanyants, A. G. Tuchin, V. A. Shishov, Ballistics-navigation support of the spacecraft flight control and implementation of "RadioAstron" scientific program. 5 years of flight, Vestnik NPO im. S.A. Lavochkina 3 (33) (2016) 25-37, in Russian.

[16] N. Ashby, Testing relativity with a laser-cooled cesium clock in space, in: Proceedings of the 1998 IEEE International Frequency Control Symposium (Cat. No.98CH36165), 1998, pp. 320-328. doi:10.1109/FREQ.1998.717923

[17] Blanchet, L., Salomon, C., Teyssandier, P., Wolf, P., Relativistic theory for time and frequency transfer to order $c^{-3}, \mathrm{~A} \& \mathrm{~A}$ 370 (1) (2001) 320-329. doi:10.1051/0004-6361:20010233 URL https://doi .org/10.1051/0004-6361:20010233

[18] Duev, D. A., Molera Calvés, G., Pogrebenko, S. V., Gurvits, L. I., Cimó, G., Bocanegra Bahamon, T., Spacecraft VLBI and Doppler tracking: algorithms and implementation A\&A 541 (2012) A43. doi:10.1051/0004-6361/201218885 URL https://doi .org/10.1051/0004-6361/201218885

[19] A. V. Gusev, D. A. Litvinov, V. N. Rudenko, et al., , to be published in Classical and Quantum Gravity (2018).

[20] N. K. Pavlis, S. A. Holmes, S. C. Kenyon, J. K. Factor, The development and evaluation of the Earth Gravitational Model 2008 (EGM2008) Journal of Geophysical Research: Solid Earth 117 (B4) (2012) n/a-n/a, b04406. doi:10.1029/2011JB008916. URL http://dx.doi.org/10.1029/2011JB008916

[21] M. Hernández-Pajares, J. M. Juan, J. Sanz, R. Orus, A. GarciaRigo, J. Feltens, A. Komjathy, S. C. Schaer, A. Krankowski, The IGS VTEC maps: a reliable source of ionospheric information since 1998, Journal of Geodesy 83 (3) (2009) 263-275. doi: $10.1007 / \mathrm{s} 00190-008-0266-1$

URL http://dx.doi .org/10.1007/s00190-008-0266-1

[22] J. Boehm, B. Werl, H. Schuh, Troposphere mapping functions for GPS and very long baseline interferometry from European Centre for Medium-Range Weather Forecasts operational analysis data, Journal of Geophysical Research: Solid Earth 111 (B2) (2006) n/a-n/a, b02406. doi:10.1029/2005JB003629 URL http://dx.doi.org/10.1029/2005JB003629

[23] T. D. Moyer, Formulation for observed and computed values of Deep Space Network data types for navigation, John Wiley \& Sons, 2005.

[24] E. Thébault, C. C. Finlay, C. D. Beggan, P. Alken, J. Aubert, 
O. Barrois, F. Bertrand, T. Bondar, A. Boness, L. Brocco,

E. Canet, A. Chambodut, A. Chulliat, P. Coïsson, F. Civet,

A. Du, A. Fournier, I. Fratter, N. Gillet, B. Hamilton,

M. Hamoudi, G. Hulot, T. Jager, M. Korte, W. Kuang,

X. Lalanne, B. Langlais, J.-M. Léger, V. Lesur, F. J. Lowes,

S. Macmillan, M. Mandea, C. Manoj, S. Maus, N. Olsen,

V. Petrov, V. Ridley, M. Rother, T. J. Sabaka, D. Saturnino,

R. Schachtschneider, O. Sirol, A. Tangborn, A. Thomson,

L. Tøffner-Clausen, P. Vigneron, I. Wardinski, T. Zvereva, International geomagnetic reference field: the 12th generation Earth, Planets and Space 67 (1) (2015) 79. doi:10.1186/ s40623-015-0228-9

URL http://dx.doi .org/10.1186/s40623-015-0228-9

[25] O. J. Sovers, J. L. Fanselow, C. S. Jacobs, Astrometry and geodesy with radio interferometry: experiments, models, results $\mid$ Rev. Mod. Phys. 70 (1998) 1393-1454. doi: 10.1103/RevModPhys.70.1393

URL https://link.aps.org/doi/10.1103/RevModPhys.70. 1393

[26] D. A. Litvinov, U. Bach, N. Bartel, K. G. Belousov, M. Bietenholz, A. V. Biriukov, G. Cimo, D. A. Duev, L. I. Gurvits, A. V. Gusev, R. Haas, V. L. Kauts, B. Z. Kanevsky, A. V. Kovalenko, G. Kronschnabl, V. V. Kulagin, M. Lindqvist, G. Molera Calves, A. Neidhardt, C. Ploetz, S. V. Pogrebenko, N. K. Porayko, V. N Rudenko, A. I. Smirnov, K. V. Sokolovsky, V. A. Stepanyants, J. Yang, M. V. Zakhvatkin, RadioAstron gravitational redshift experiment: status update, ArXiv e-printsarXiv:1605.05832

[27] M. Pearlman, J. Degnan, J. Bosworth, The international laser ranging service, Advances in Space Research 30 (2) (2002) 135 - 143. doi:http://dx.doi.org/10.1016/S0273-1177(02) 00277-6.

URL http://www.sciencedirect.com/science/article/pii/ S0273117702002776 